\title{
PENGGUNAAN METODE MITTAG-LEFFLER UNTUK MENYELESAIKAN PERSAMAAN DIFERENSIAL FRAKSIONAL LINIER DENGAN KOEFISIEN KONSTAN
}

\author{
GUSTI NELLAM SARI, AHMAD IQBAL BAQI \\ Program Studi S1 Matematika, \\ Fakultas Matematika dan Ilmu Pengetahuan Alam, Universitas Andalas, \\ Kampus UNAND Limau Manis Padang, Indonesia. \\ email : gnellamsari@gmail.com,6baqi@sci.unand.ac.id

Abstrak. Dalam makalah ini digunakan metode Mittag-Leffler berikut:

$$
y(x)=E_{\alpha}\left(a x^{\alpha}\right)=\sum_{n=0}^{\infty} a^{n} \frac{x^{n \alpha}}{\Gamma(n \alpha+1)},
$$

untuk menyelesaikan beberapa tipe persamaan diferensial fraksional dengan koefisien konstan. Beberapa contoh diberikan untuk mengilustrasikan hasil utama.

Kata Kunci: Persamaan Diferensial Fraksional, Turunan Fraksional Caputo, Fungsi Mittag-Leffler

\section{Pendahuluan}

Persamaan diferensial muncul dalam berbagai macam model matematika di bidang sains dan teknologi. Persamaan diferensial adalah persamaan yang memuat suatu fungsi tak diketahui beserta turunan-turunannya.

Suatu persamaan diferensial fraksional adalah suatu persamaan diferensial dengan orde berupa bilangan rasional. Persamaan diferensial fraksional pertama kali diperkenalkan oleh L'Hopital pada tahun 1695 seiring dengan perkembangan kalkulus fraksional.

Penelitian untuk mendapatkan solusi persamaan diferensial fraksional telah banyak dilakukan. Dalam penelitian ini akan dicari solusi beberapa tipe persamaan diferensial fraksional linier homogen dengan koefisien konstan menggunakan metode Mittag-Leafler:

$$
y(x)=E_{\alpha}\left(a x^{\alpha}\right)=\sum_{n=0}^{\infty} a^{n} \frac{x^{n \alpha}}{\Gamma(n \alpha+1)} .
$$

${ }^{*}$ penulis korespondensi 


\section{Landasan Teori}

\subsection{Fungsi Gamma}

Definisi 2.1. [5] Fungsi Gamma dinotasikan dengan $\Gamma(n)$, didefinisikan sebagai

$$
\Gamma(n)=\int_{0}^{\infty} t^{n-1} e^{-t} d t, n>0, n \in \mathbb{R} .
$$

\subsection{Fungsi Beta}

Definisi 2.2. [3] Fungsi Beta dinotasikan B( $p, q)$, didefinisikan sebagai berikut:

$$
B(p, q)=\int_{0}^{1} x^{p-1}(1-x)^{q-1} d x, x \in \mathbb{R},
$$

$\operatorname{dimana} \operatorname{Re}(p)>0$ dan $\operatorname{Re}(q)>0$.

\subsection{Fungsi Mittag-Leffler dan Turunan Fraksional Caputo}

Definisi 2.3. [1] Fungsi Mittag-Leffler satu parameter $(\alpha)$ dinotasikan dengan $E_{\alpha}$, didefinisikan sebagai berikut:

$$
E_{\alpha}(z)=\sum_{k=0}^{\infty} \frac{z^{k}}{\Gamma(\alpha k+1)}, z \in \mathbb{C} .
$$

Definisi 2.4. [2] Turunan fraksional Caputo orde $\alpha \in \mathbb{R}$ dari fungsi $y(x)$ dengan $m-1<\alpha<m, m \in \mathbb{N}$, didefinisikan sebagai berikut

$$
D^{\alpha} y(x)=\frac{1}{\Gamma(m-\alpha)} \int_{0}^{x} \frac{y^{(m)}(\tau) d \tau}{(x-\tau)^{\alpha+1-m}},
$$

dimana $y^{(m)}(\tau)$ adalah turunan ke- $m$ dari fungsi $y(\tau)$.

[4] Perumuman dari metode Mittag-Leffler disajikan

$$
y=E_{\alpha}\left(a x^{\alpha}\right)=\sum_{n=0}^{\infty} a^{n} \frac{x^{n \alpha}}{\Gamma(n \alpha+1)} .
$$

Selanjutnya, gunakan Definisi dari kalkulus fraksional sebagai berikut:

$$
\begin{gathered}
D^{\alpha} y(x)=\sum_{n=1}^{\infty} a^{n} \frac{x^{(n-1) \alpha}}{\Gamma((n-1) \alpha+1)} . \\
D^{(2 \alpha)} y(x)=\sum_{n=2}^{\infty} a^{n} \frac{x^{(n-2) \alpha}}{\Gamma((n-2) \alpha+1)} . \\
D^{(3 \alpha)} y(x)=\sum_{n=3}^{\infty} a^{n} \frac{x^{(n-3) \alpha}}{\Gamma((n-3) \alpha+1)} .
\end{gathered}
$$




$$
\begin{gathered}
D^{(m-1) \alpha} y(x)=\sum_{n=m-1}^{\infty} a^{n} \frac{x^{(n-(m-1)) \alpha}}{\Gamma((n-(m-1)) \alpha+1)} \\
D^{(m \alpha)} y(x)=\sum_{n=m}^{\infty} a^{n} \frac{x^{(n-m) \alpha}}{\Gamma((n-m) \alpha+1)}
\end{gathered}
$$

\section{Pembahasan}

Berikut ini diperlihatkan bagaimana metoda Mittag Leafler digunakan untuk menyelesaikan beberapa tipe persamaan diferensial fraksional linier dengan koefisien konstan.

(1) $\frac{d^{\alpha} y}{d x^{\alpha}}=k y$.

Dengan mensubstitusikan persamaan (2.1) diperoleh

$$
\begin{aligned}
\sum_{n=1}^{\infty} a^{n} \frac{x^{(n-1) \alpha}}{\Gamma((n-1) \alpha+1)}-k \sum_{n=0}^{\infty} a^{n} \frac{x^{n \alpha}}{\Gamma(n \alpha+1)} & =0 \\
\sum_{n=0}^{\infty} a^{n+1} \frac{x^{n \alpha}}{\Gamma((n \alpha+1)}-k \sum_{n=0}^{\infty} a^{n} \frac{x^{n \alpha}}{\Gamma(n \alpha+1)} & =0 \\
\sum_{n=0}^{\infty}\left(a^{n+1}-k a^{n}\right) n \frac{x^{n \alpha}}{\Gamma(n \alpha+1)} & =0 .
\end{aligned}
$$

Dengan menjadikan koefisien dari $x^{n \alpha}$ sama dengan 0 , diperoleh

$$
a^{n+1}-k a^{n}=0 .
$$

untuk $n=0$, maka diperoleh $a^{1}=k a^{0}=k$.

untuk $n=1$, maka diperoleh $a^{2}=k a^{1} \Rightarrow a^{2}=k^{2}$, untuk $n=2$, maka diperoleh $a^{3}=k a^{2} \Rightarrow a^{3}=k^{3}$.

Berikutnya substitusikan ke persamaan (1.1)

$$
\begin{aligned}
& y(x)=a^{0}+a^{1} \frac{x^{a}}{\Gamma(\alpha+1)}+a^{2} \frac{x^{2 \alpha}}{\Gamma(2 \alpha+1)}+a^{3} \frac{x^{3 \alpha}}{\Gamma(3 \alpha+1)}+\cdots \\
& y(x)=1+k \frac{x^{a}}{\Gamma(\alpha+1)}+k^{2} \frac{x^{2 \alpha}}{\Gamma(2 \alpha+1)}+k^{3} \frac{x^{3 \alpha}}{\Gamma(3 \alpha+1)}+\cdots
\end{aligned}
$$

Solusi umum adalah

$$
y(x)=\sum_{n=0}^{\infty} \frac{k^{n} x^{n \alpha}}{\Gamma(n \alpha+1)} .
$$

Dengan $\alpha=1$, solusi eksaknya adalah:

$$
y(x)=\sum_{n=0}^{\infty} \frac{(k x)^{n}}{\Gamma(n+1)} .
$$


(2) $\frac{d^{2 \alpha} y}{d x^{2 \alpha}}-y=0$.

Dengan mensubstitusi persamaan (2.2) diperoleh

$$
\begin{aligned}
\sum_{n=2}^{\infty} a^{n} \frac{x^{(n-2) \alpha}}{\Gamma((n-2) \alpha+1)}-\sum_{n=0}^{\infty} a^{n} \frac{x^{n \alpha}}{\Gamma(n \alpha+1)} & =0 \\
\sum_{n=0}^{\infty} a^{n+2} \frac{x^{n \alpha}}{\Gamma(n \alpha+1)}-\sum_{n=0}^{\infty} a^{n} \frac{x^{n \alpha}}{\Gamma(n \alpha+1)} & =0, \\
\sum_{n=0}^{\infty}\left(a^{n+2}-a^{n}\right) \frac{x^{n \alpha}}{\Gamma(n \alpha+1)} & =0 .
\end{aligned}
$$

Dengan koefisien dari $x^{n \alpha}=0$, dan identifikasi koefisien sehingga diperoleh

$$
a^{n+2}=a^{n} .
$$

Selanjutnya substitusikan ke persamaan (1.1)

$$
y(x)=1+a \frac{x^{\alpha}}{\Gamma(\alpha+1)}+\frac{x^{2 \alpha}}{\Gamma(2 \alpha+1)}+a \frac{x^{3 \alpha}}{\Gamma(3 \alpha+1)}+\cdots
$$

Jika $a=1$, maka solusi umum dalam fungsi Mittag-Leffler

$$
y(x)=\sum_{n=0}^{\infty} \frac{x^{n \alpha}}{\Gamma(n \alpha+1)}=E_{\alpha}\left(x^{\alpha}\right) .
$$

(3) $\frac{d^{2 \alpha} y}{d x^{2 \alpha}}+\frac{d^{\alpha} y}{d x^{\alpha}}-2 y=0$.

Dengan mensubstitusi persamaan (2.2) diperoleh

$$
\begin{aligned}
\sum_{n=2}^{\infty} a^{n} \frac{x^{(n-2) \alpha}}{\Gamma((n-2) \alpha+1)}+\sum_{n=1}^{\infty} a^{n} \frac{x^{(n-1) \alpha}}{\Gamma((n-1) \alpha+1)}-2 \sum_{n=0}^{\infty} a^{n} \frac{x^{(n \alpha)}}{\Gamma(n \alpha+1)} & =0 . \\
\sum_{n=0}^{\infty} a^{n+2} \frac{x^{n \alpha}}{\Gamma(n \alpha+1)}+\sum_{n=0}^{\infty} a^{n+1} \frac{x^{n \alpha}}{\Gamma(n \alpha+1)}-2 \sum_{n=0}^{\infty} a^{n} \frac{x^{(n \alpha)}}{\Gamma(n \alpha+1)} & =0 . \\
\sum_{n=0}^{\infty}\left(a^{n+2}+a^{n+1}-2 a^{n}\right) \frac{x^{n \alpha}}{\Gamma(n \alpha+1)} & =0 .
\end{aligned}
$$

Dengan koefisien $x^{n \alpha}=0$, dan identifikasi koefisien sehingga,

$$
a^{n+2}=2 a^{n}-a^{n+1} .
$$

Sehingga diperoleh:

$$
y(x)=1+a \frac{x^{\alpha}}{\Gamma(\alpha+1)}+(2-a) \frac{x^{2 \alpha}}{\Gamma(2 \alpha+1)}+a(2-a) \frac{x^{3 \alpha}}{\Gamma(3 \alpha+1)}+\cdots
$$

Jika $a=1$, maka solusi umum dari fungsi Mittag-Leffler

$$
y(x)=\sum_{n=0}^{\infty} \frac{x^{n \alpha}}{\Gamma(n \alpha+1)}=E_{\alpha}\left(x^{\alpha}\right) .
$$


(4) $\frac{d^{2 \alpha} y}{d x^{2 \alpha}}+a_{1} \frac{d^{\alpha} y}{d x^{\alpha}}+a_{o} y=0$.

Dengan mensubstitusi persamaan (2.2) diperoleh

$$
\begin{array}{r}
\sum_{n=2}^{\infty} a^{n} \frac{x^{(n-2) \alpha}}{\Gamma((n-2) \alpha+1)}+a_{1} \sum_{n=1}^{\infty} a^{n} \frac{x^{(n-1) \alpha}}{\Gamma((n-1) \alpha+1)}+a_{0} \sum_{n=0}^{\infty} a^{n} \frac{x^{(n \alpha)}}{\Gamma(n \alpha+1)}=0 . \\
\sum_{n=0}^{\infty} a^{n+2} \frac{x^{n \alpha}}{\Gamma(n \alpha+1)}+a_{1} \sum_{n=0}^{\infty} a^{n+1} \frac{x^{n \alpha}}{\Gamma(n \alpha+1)}+a_{0} \sum_{n=0}^{\infty} a^{n} \frac{x^{(n \alpha)}}{\Gamma(n \alpha+1)}=0 . \\
\sum_{n=0}^{\infty}\left(a^{n+2}+a_{1} a^{n+1}+a_{0} a^{n}\right) \frac{x^{n \alpha}}{\Gamma(n \alpha+1)}=0 .
\end{array}
$$

Dengan menjadikan koefisien dari $x^{n \alpha}$ sama dengan 0, diperoleh:

$$
\begin{aligned}
a^{n+2}+a_{1} a^{n+1}+a_{0} a^{n} & =0 . \\
a^{n+2} & =-a_{1} a^{n+1}-a_{0} a^{n} .
\end{aligned}
$$

untuk $n=0$, maka diperoleh

$$
a^{2}=-a_{1} a-a_{0} .
$$

untuk $n=1$, maka diperoleh

$$
a^{3}=-a_{1} a^{2}-a_{0} a=a\left(-a_{1} a-a_{0}\right) .
$$

untuk $n=2$, maka diperoleh

$$
a^{4}=-a_{1} a^{3}-a_{0} a^{2}=a^{2}\left(-a_{1} a-a_{0}\right) .
$$

Substitusi nilai yang diperoleh pada persamaan (3.2), (3.3), dan (3.4) ke persamaan (1.1). Selanjutnya diperoleh hasil

$$
y(x)=1+a \frac{x^{\alpha}}{\Gamma(\alpha+1)}+\left(-a_{1} a-a_{0}\right) \frac{x^{2 \alpha}}{\Gamma(2 \alpha+1)}+a\left(-a_{1} a-a_{0}\right) \frac{x^{3 \alpha}}{\Gamma(3 \alpha+1)}+\cdots
$$

Dengan pemisalan $a^{2}$ pada persamaan (3.2), maka persamaan (3.5) dapat disederhanakan menjadi

$$
y(x)=1+a \frac{x^{\alpha}}{\Gamma(\alpha+1)}+a^{2} \frac{x^{2 \alpha}}{\Gamma(2 \alpha+1)}+a^{3} \frac{x^{3 \alpha}}{\Gamma(3 \alpha+1)}+\cdots
$$

Jika $a=1$, maka solusi umum dari fungsi Mittag-Leffler

$$
y(x)=\sum_{n=0}^{\infty} \frac{x^{n \alpha}}{\Gamma(n \alpha+1)}=E_{\alpha}\left(x^{\alpha}\right) .
$$


(5) $D^{3 \alpha} y+\alpha_{2} D^{2 \alpha} y+\alpha_{1} D^{2} y+\alpha_{0} y=0$.

Dengan mensubstitusi persamaan (2.3) diperoleh

$$
\begin{aligned}
& \sum_{n=3}^{\infty} a^{n} \frac{x^{(n-3) \alpha}}{\Gamma((n-3) \alpha+1)}+\alpha_{2} \sum_{n=2}^{\infty} a^{n} \frac{x^{(n-2) \alpha}}{\Gamma((n-2) \alpha+1)} \\
& +\alpha_{1} \sum_{n=1}^{\infty} a^{n} \frac{x^{(n-1) \alpha}}{\Gamma(n-1 \alpha+1)}+\alpha_{0} \sum_{n=0}^{\infty} \frac{x^{n \alpha}}{\Gamma(n \alpha+1)}=0 \\
& \sum_{n=0}^{\infty} a^{n+3} \frac{x^{n \alpha}}{\Gamma(n \alpha+1)}+\alpha_{2} \sum_{n=0}^{\infty} a^{n+2} \frac{x^{n \alpha}}{\Gamma n \alpha+1)} \\
& +\alpha_{1} \sum_{n=0}^{\infty} a^{n+1} \frac{x^{n \alpha}}{\Gamma(n \alpha+1)}+\alpha_{0} \sum_{n=0}^{\infty} \frac{x^{n \alpha}}{\Gamma(n \alpha+1)}=0 . \\
& \sum_{n=0}^{\infty}\left(a^{n+3}+\alpha_{2} a^{n+2}+\alpha_{1} a^{n+1}+\alpha_{0}\right) \frac{x^{n \alpha}}{\Gamma(n \alpha+1)}=0 .
\end{aligned}
$$

Dengan menjadikan koefisien dari $x^{n \alpha}$ sama dengan 0, diperoleh

$$
\begin{aligned}
a^{n+3}+\alpha_{2} a^{n+2}+\alpha_{1} a^{n+1}+\alpha_{0} a^{n} & =0 . \\
a^{n+3} & =-\alpha_{2} a^{n+2}-\alpha_{1} a^{n+1}-\alpha_{0} a^{n} .
\end{aligned}
$$

untuk $n=0$, maka diperoleh

$$
a^{3}=-\alpha_{2} a^{2}-\alpha_{1} a^{1}-\alpha_{0} .
$$

untuk $n=1$, maka diperoleh

$$
a^{4}=-\alpha_{2} a^{3}-\alpha_{1} a^{2}-\alpha_{0} a^{1} .=a\left(-\alpha_{2} a^{2}-\alpha_{1} a^{1}-\alpha_{0}\right) .
$$

untuk $n=2$, maka diperoleh

$$
a^{5}=-\alpha_{2} a^{4}-\alpha_{1} a^{3}-\alpha_{0} a^{2}=a^{2}\left(-\alpha_{2} a^{2}-\alpha_{1} a^{1}-\alpha_{0}\right) .
$$

Substitusi nilai yang diperoleh pada persamaan (3.8), (3.9) dan (3.10) ke persamaan (1.1). Selanjutnya diperoleh hasil

$y(x)=1+a \frac{x^{\alpha}}{\Gamma(\alpha+1)}+a^{2} \frac{x^{2 \alpha}}{\Gamma(2 \alpha+1)}+\left(-\alpha_{2} a^{2}-\alpha_{1} a^{1}-\alpha_{0}\right) \frac{x^{3 \alpha}}{\Gamma(3 \alpha+1)}+\cdots$

Dengan pemisalan $a^{3}$ pada persamaan (3.8), maka persamaan (3.11) dapat disederhanakan menjadi

$$
y(x)=1+a \frac{x^{\alpha}}{\Gamma(\alpha+1)}+a^{2} \frac{x^{2 \alpha}}{\Gamma(2 \alpha+1)}+a^{3} \frac{x^{3 \alpha}}{\Gamma(3 \alpha+1)}+\cdots
$$

Jika $a=1$, maka solusi umum dari fungsi Mittag-Leffler

$$
y(x)=\sum_{n=0}^{\infty} \frac{x^{n \alpha}}{\Gamma(n \alpha+1)}=E_{\alpha}\left(x^{\alpha}\right) .
$$


(6) $D^{m \alpha} y+\alpha_{m-1} D^{(m-1) \alpha} y+\alpha_{m-2} D^{(m-2) \alpha} y+\ldots+\alpha_{1} D^{\alpha} y+\alpha_{0} y=0$.

Dengan mensubstitusi persamaan (2.4) diperoleh

$$
\begin{aligned}
& \sum_{n=m}^{\infty} a^{n} \frac{x^{(n-m) \alpha}}{\Gamma((n-m) \alpha+1)}+\alpha_{m-1} \sum_{n=m-1}^{\infty} a^{n} \frac{x^{(n-(m-1) \alpha}}{\Gamma((n-(m-1) \alpha+1)} . \\
& +\alpha_{m-2} \sum_{n=m-2}^{\infty} a^{n} \frac{x^{(n-(m-2) \alpha}}{\Gamma((n-(m-1) \alpha+1)}+\ldots+\alpha_{1} \sum_{n=1}^{\infty} a^{n} \frac{x^{(n-1) \alpha}}{\Gamma((n-1) \alpha+1)} \\
& +\alpha_{0} \sum_{n=0}^{\infty} a^{n} \frac{x^{(n \alpha)}}{\Gamma(n \alpha+1)}=0 . \\
& \quad \sum_{n=0}^{\infty} a^{(n+m)} \frac{x^{(n \alpha)}}{\Gamma(n \alpha+1)}+\alpha_{m-1} \sum_{n=0}^{\infty} a^{n+m-1} \frac{x^{(n \alpha)}}{\Gamma(n \alpha+1)} \\
& \quad+\alpha_{m-2} \sum_{n=0}^{\infty} a^{(n+m-2)} \frac{x^{(n \alpha)}}{\Gamma(n \alpha+1)}+\cdots+\alpha_{1} \sum_{n=0}^{\infty} a^{n+1} \frac{x^{(n \alpha)}}{\Gamma(n \alpha+1)} \\
& \quad+\alpha_{0} \sum_{n=0}^{\infty} a^{n} \frac{x^{(n \alpha)}}{\Gamma(n \alpha+1)}=0 . \\
& \quad \sum_{n=0}^{\infty}\left(a^{(n+m)}+\alpha_{(m-1)} a^{(n+m-1)}+\alpha_{(m-2)} a^{(n+m-2)}+\ldots+\alpha_{1} a^{(n+1)}\right. \\
& +\alpha_{0} a^{n} \frac{x^{n \alpha}}{\Gamma(n \alpha+1)}=0
\end{aligned}
$$

Dengan menjadikan koefisien dari $x^{n \alpha}$ sama dengan 0, diperoleh

$$
\begin{gathered}
\left(a^{(n+m)}+\alpha_{(m-1)} a^{(n+m-1)}+\alpha_{(m-2)} a^{(n+m-2)}+\ldots+\alpha_{1} a^{(n+1)}+\alpha_{0} a^{n}\right)=0 \\
\left(a^{(n+m)}=-\alpha_{(m-1)} a^{(n+m-1)}-\alpha_{(m-2)} a^{(n+m-2)}-\ldots-\alpha_{1} a^{(n+1)}-\alpha_{0} a^{n}\right)
\end{gathered}
$$

untuk $n=0$, maka diperoleh

$$
\left.a^{m}=-\alpha_{(m-1)} a^{(m-1)}-\alpha_{(m-2)} a^{(m-2)}-\ldots-\alpha_{1} a-\alpha_{0}\right)
$$

untuk $n=1$, maka diperoleh

$$
\begin{aligned}
a^{m+1} & \left.=-\alpha_{(m-1)} a^{m}-\alpha_{(m-2)} a^{(m-1)}-\ldots-\alpha_{1} a^{2}-\alpha_{0} a\right) \\
& =a\left(-\alpha_{(m-1)} a^{(m-1)}-\alpha_{(m-2)} a^{(m-2)}-\ldots-\alpha_{1} a-\alpha_{0}\right)
\end{aligned}
$$

untuk $n=2$, maka diperoleh

$$
\begin{aligned}
a^{m+2} & \left.=-\alpha_{(m-1)} a^{m+1}-\alpha_{(m-2)} a^{m}-\ldots-\alpha_{1} a^{3}-\alpha_{0} a^{2}\right) \\
& =a^{2}\left(-\alpha_{(m-1)} a^{(m-1)}-\alpha_{(m-2)} a^{(m-2)}-\ldots-\alpha_{1} a-\alpha_{0}\right)
\end{aligned}
$$

untuk $n=3$, maka diperoleh

$$
\begin{aligned}
a^{m+3} & \left.=-\alpha_{(m-1)} a^{m+2}-\alpha_{(m-2)} a^{m+1}-\ldots-\alpha_{1} a^{4}-\alpha_{0} a^{3}\right) \\
& =a^{3}\left(-\alpha_{(m-1)} a^{(m-1)}-\alpha_{(m-2)} a^{(m-2)}-\ldots-\alpha_{1} a-\alpha_{0}\right)
\end{aligned}
$$


Substitusikan persamaan (3.13), (3.14), (3.15), dan (3.16) ke persamaan (1.1) diperoleh

$$
y(x)=1+a \frac{x^{\alpha}}{\Gamma(\alpha+1)}+a^{2} \frac{x^{2 \alpha}}{\Gamma(2 \alpha+1)}+a^{3} \frac{x^{3 \alpha}}{\Gamma(3 \alpha+1)}+\ldots
$$

Jika $a=1$, maka solusi umum dari fungsi Mittag-Leffler

$$
y(x)=\sum_{n=0}^{\infty} \frac{x^{n \alpha}}{\Gamma(n \alpha+1)}=E_{\alpha}\left(x^{\alpha}\right)
$$

\section{Kesimpulan}

Metode Mittag-Leffler adalah salah satu metode yang dapat menyelesaikan beberapa tipe persamaan diferensial fraksional linier dengan koefisien konstan.

\section{Ucapan Terima kasih}

Terima kasih kepada bapak Muhafzan, ibu Nova Noliza Bakar, bapak Zulakmal, dan ibu Riri Lestari yang telah memberikan kritik dan saran untuk penulisan artikel ini

\section{Daftar Pustaka}

[1] I. Podlubny, Fractional Differential Equation: An Introduction to Fractional Derivatives, Fractional Differential Equation,Some Metods of Their Solution and Some Their Applications to Methods of their Solution and Some of Their Applications, Academic Press, New York, NY, USA, 1999.

[2] Kaczorek, T. 2011. Selected Problem of Fractional System Theory SpringerVerlag, Berlin Heidelberg.

[3] Milici. Constantin, Gheorghe. D, J. Tanreiro. 2019. to Fractional Diffe-rential Equations. Springer, Switzerland.

[4] Rida, S. Z dan A. A. M. Arafa . 2011. New Method for Solving Linier Fractional Differential Equation, International Journal of Differential Equation, Article ID 814132

[5] Spiegel, M. R. 2002. Theory and Problems of Advanced Calculus. McGraw-Hill International Book Company: New York. 